University of Nebraska - Lincoln

DigitalCommons@University of Nebraska - Lincoln

May 1993

\title{
Experimental determination of the unoccupied bands of W(110)
}

Dongqi Li

University of Nebraska-Lincoln and the Syracuse University

Peter A. Dowben

University of Nebraska-Lincoln, pdowben@unl.edu

J.E. Ortega

IBM Thomas J. Watson Research Center, Yorktown Heights, New York

F.J. Himpsel

IBM Thomas J. Watson Research Center, Yorktown Heights, New York

Follow this and additional works at: https://digitalcommons.unl.edu/physicsdowben

Part of the Physics Commons

Li, Dongqi; Dowben, Peter A.; Ortega, J.E.; and Himpsel, F.J., "Experimental determination of the unoccupied bands of W(110)" (1993). Peter Dowben Publications. 59.

https://digitalcommons.unl.edu/physicsdowben/59

This Article is brought to you for free and open access by the Research Papers in Physics and Astronomy at DigitalCommons@University of Nebraska - Lincoln. It has been accepted for inclusion in Peter Dowben Publications by an authorized administrator of DigitalCommons@University of Nebraska - Lincoln. 


\title{
Experimental determination of the unoccupied bands of W(110)
}

\author{
Dongqi Li and P. A. Dowben \\ Department of Physics, Syracuse University, Syracuse, New York 13244-1130 \\ J. E. Ortega and F. J. Himpsel \\ IBM Thomas J. Watson Research Center, Yorktown Heights, New York 10598
}

(Received 8 October 1992)

\begin{abstract}
Inverse photoemission from $\mathrm{W}(110)$ has been used to determine the dispersion of the unoccupied energy bands along the tungsten $\Gamma \Sigma N$ line. The critical points are determined to be $\Gamma_{12}$ at $2.6 \mathrm{eV}$, $N_{1}$ at $2.2 \mathrm{eV}$, and the lower limit of $N_{4}$ at $3.2 \mathrm{eV}$. The upper bound of the lifetime broadening is estimated as $0.3-0.4 \mathrm{eV}$ at $2-3 \mathrm{eV}$ above $E_{F}$. The image state is observed at $4.5 \mathrm{eV}$ above $E_{F}$, or $0.7 \mathrm{eV}$ in binding energy, relative to the vacuum level.
\end{abstract}

\section{INTRODUCTION}

The band structure of tungsten has been studied extensively ${ }^{1-17}$ for a variety of reasons. Tungsten is a useful high-temperature material, and is used in fieldemission- and scanning-tunneling-microscope tips. In addition, tungsten serves as a convenient substrate for the growth of epitaxial ferromagnetic-rare-earth films. The W(100) surface exhibits an unusual reconstruction, ${ }^{8,18}$ but the W(110) surface is more stable and therefore suitable for bulk-band-structure studies. While the occupied part of the band structure has been extensively investigated with photoemission for different surfaces, ${ }^{9-12}$ the unoccupied bands have not been fully characterized experimentally. ${ }^{14-17}$ The previous measurements of empty bands with inverse-photoemission were limited and did not map out the band dispersion experimentally.

We used a high-resolution, tunable inverse-photoemission system to map the unoccupied bands at the close-packed W(110) surface. In the following, we will show the experimental dispersion of the bulk bands along the $\Gamma \Sigma N$ line, and compare our results with the previously published experimental data and the band calculations. The experimental results for the unoccupied $W$ $5 d \Sigma_{1}$ and $\Sigma_{4}$ bands reveal that the dispersion is qualitatively similar to that obtained by theoretical calculations but are shifted to the higher energies by some $0.1-0.7 \mathrm{eV}$ for different parts of the bands. The real and imaginary parts of the self-energy will be discussed. We find an image potential surface state at $4.5 \mathrm{eV}$ that has not been previously reported.

\section{EXPERIMENT}

The experiment was carried out on a high-resolution inverse-photoemission spectrometer with a photon energy range of $8-30 \mathrm{eV}$ and up to $44 \mathrm{eV}$ in second order. The energy resolution was $0.26 \mathrm{eV}$ [half-width at full maximum (HWFM)] at $9 \mathrm{eV}$ and was larger at higher energies $(0.6 \mathrm{eV}$ at $18 \mathrm{eV})$. The accuracy of the energy calibration is $0.1 \mathrm{eV}$. All the spectra in this work were taken at room temperature. The pressure of the spectrometer chamber was in the $10^{-11}$-torr range. The $\mathrm{W}(110)$ single crystal has been cleaned with the normal procedure (oxygen treatment followed by flashing) ${ }^{19}$ in the attached preparation chamber. The surface cleanliness and order of the crystal after such a procedure were checked with photoemission and low-energy electron diffraction (LEED) in another separate chamber, and with LEED in the preparation chamber.

\section{RESULTS}

Figure 1 shows a typical inverse-photoemission spectrum with an initial electron energy of $14.5 \mathrm{eV}$. The feature at $4.54 \mathrm{eV}$ is very sensitive to contamination and shows no $k_{\perp}$ dependence. We interpret this feature as the image state of $W(110)$. Taking the work function of $\mathrm{W}(110)$ as $5.25 \mathrm{eV}$, the binding energy of this state (energy below the vacuum level) is $0.7 \mathrm{eV}$, the same as that observed for W(001) (Ref. 8) within the experimental error. The appearance of this image state is an excellent indication that the surface is contamination-free. The three features at lower energies exhibit energy dispersion (relative to $E_{F}$ ) with the incident electron energy (dependence upon $k_{\perp}$ ). From a comparison with existing band-structure calculations, we can identify these features as the bulk bands $\Sigma_{1}, \Sigma_{1}$, and $\Sigma_{4}$, respectively (see Fig. 1).

In order to determine the value of $k_{\perp}$ experimentally, we measured the intensity at $E_{F}$ for the different initial electron energies (Fig. 2). It is clear that there are two Fermi-level crossing points at $11.5 \mathrm{eV}$ and $20.5 \mathrm{eV}$. According to the existing de Haas-van Alphen ( $\mathrm{dHvA}$ ) data, ${ }^{20}$ Fermi-level crossings should occur at wave vectors of $0.09(N \Gamma)$ and $0.67(N \Gamma)$, respectively. Based on these two experimentally determined points of the initial band, we can assign $k_{\perp}$ values as noted below. We take a free-electron parabola with an inner potential of -0.63 $\mathrm{eV}$ relative to the Fermi level to account for the higher energy part of the initial band. The $\Gamma$ point is reached 


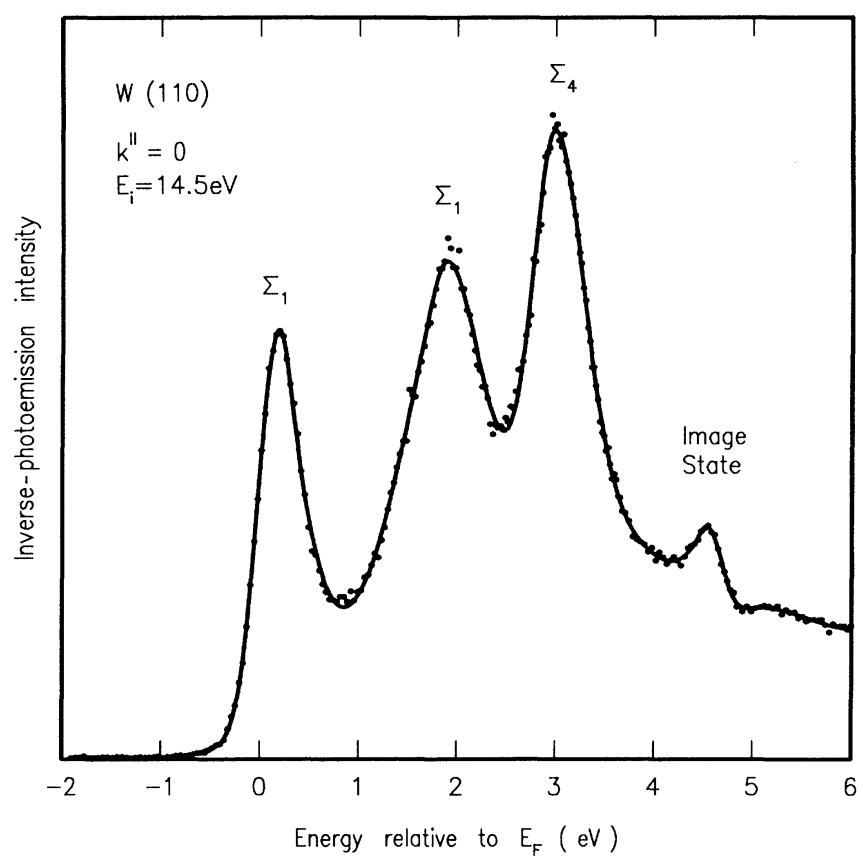

FIG. 1. A typical inverse-photoemission spectrum at normal incidence on the W(110) surface. The image state and the band assignments are indicated in the figure.

with an electron impact energy of $29.7 \mathrm{eV}$. For the lower energy part of the initial band (near the $N$ point), a relativistic band calculation ${ }^{2}$ was used to reflect the gap near the Brillioun-zone boundary. This assignment of incidence electron energies to $k_{\perp}$ values is shown in the comparison of the upper and lower abscissas of Fig. 3.

This assignment of $k_{\perp}$ to electron kinetic energies is also supported by other evidence. Similar plots of intensity (at the fixed energies above $E_{F}$ ) versus incident electron energies also indicate when the $\Sigma_{1}$ and $\Sigma_{3}$ bands disperse through at a particular $k_{\perp}$ point. Also, a minimum width of the principal inverse-photoemission (IPE) feature (the combination of the flat $\Sigma_{1}$ and $\Sigma_{4}$ bands, unresolved at high energies) is reached around $30 \mathrm{eV}$, which confirms our assignment of the critical point $\Gamma_{12}$ (at $29.7 \mathrm{eV}$ ). Our $k_{\perp}$ assignment is therefore accurate within $\pm 5 \%$.

Figure 3 shows the experimentally determined band dispersion of the unoccupied bands of $\mathrm{W}(110)$ using the initial band as described above. For the $\langle 110\rangle$ direction of a bcc crystal, the allowed final states in dipole transitions are $\Sigma_{1}, \Sigma_{3}$, and $\Sigma_{4}$, owing to the fact that the initial state of normal incident electrons should be $\Sigma_{1}$ and no light polarization detection is made in the current setup. In Fig. 3, we mapped out the relatively flat bands of $\Sigma_{1}$ and $\Sigma_{4}$ with an energy accuracy of $0.1-0.2 \mathrm{eV}$ and momentum accuracy of $5 \%$. By averaging the split $\Sigma_{1}$ and $\Sigma_{4}$ bands near $\Gamma$, the critical point $\Gamma_{12}$ is determined as $2.6 \pm 0.2$ eV. By extrapolating the experimental points near $N$, the $N_{1}$ point is determined as $2.2 \pm 0.2 \mathrm{eV}$, and the lower limit of $N_{4}$ as $3.2 \mathrm{eV}$. Compared with a relativistic pseudopotential band calculation, ${ }^{3}$ the experimental bands

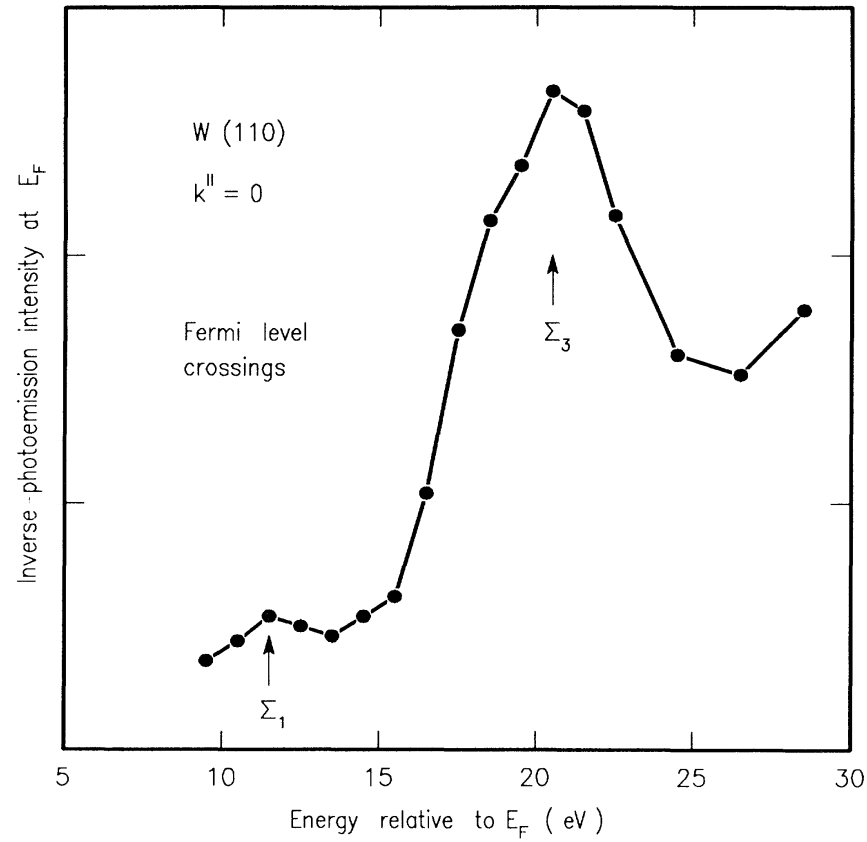

FIG. 2. Normalized intensities at $E_{F}$ of a set of inversephotoemission spectra at normal incidence, taken with different initial electron energies. The peaks at 11.5 and $20.5 \mathrm{eV}$ indicate the $\Sigma_{1}$ and $\Sigma_{3}$ bands, respectively, dispersing through $E_{F}$.

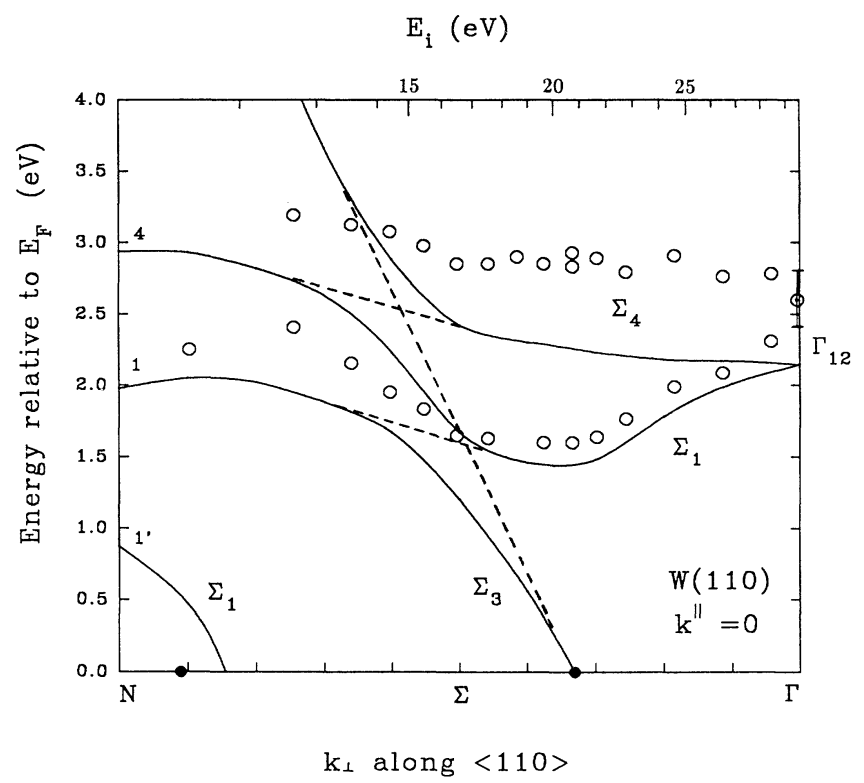

FIG. 3. Unoccupied band dispersion (open circles) along $\Gamma \Sigma N$ of $\mathrm{W}(110)$, derived from inverse-photoemission spectra. The solid circles are from dHvA data (Ref. 19), which were used to determine the $k_{\perp}$ as described in the text. The corresponding initial electron energies for different $k_{\perp}$ are shown on the upper horizontal axis. A relativistic band calculation (Ref. 3) is given for the solid lines for comparison. The band assignment is given for the nonrelativistic limit, which is shown as the dashed lines. 
are shifted to higher energies away from $E_{F}$. The significant broadening makes it difficult to map out steep bands, such as the $\Sigma_{1}$ and $\Sigma_{3}$ bands that cross $E_{F}$. We attribute the feature near $E_{F}$ to the rapidly dispersing $\Sigma_{1}$ and $\Sigma_{3}$ bands.

\section{DISCUSSION}

The unoccupied states of W(110) were previously studied with inverse photoemission by Collins et al. ${ }^{14}$ and Funnemann and Merz. ${ }^{15}$ The latter study presented very similar spectra to those reported here, although all their energy positions were shifted to higher energies by about $1 \mathrm{eV}$, possibly due to an uncertainty in the position of the Fermi level. Collins et $a l^{14}$ showed a sequence of IPE spectra with photon energies of $15-30 \mathrm{eV}$, but observed no image state and their features were less pronounced. They compared their data with photocurrent calculations, yet no attempt of a direct band mapping was made because of the difficulty of determining $k_{\perp}$. In the current work, with the experimentally determined $k_{\perp}$ and better spectral resolution, we have demonstrated that the standard three-step model of the photoemission process can still be used to understand the band structure of tungsten.

As seen in Fig. 3, there is clearly a discrepancy between the theoretical band structure and our experimental results, especially for the $\Sigma_{4}$ band. The experimentally determined $\Sigma_{4}$ band is shifted upwards for about $0.5-0.6$ $\mathrm{eV}$. At the critical point $\Gamma_{12}$, this shift is $0.5 \pm 0.2 \mathrm{eV}$. Several different types of band calculations, such as the relativistic augmented-plane-wave (RAPW) calculation done by Christensen and Feuerbacher, ${ }^{1}$ and the semirelativistic linearized APW calculations done by Jansen and Freeman $^{4}$ and Wei, Krakauer, and Weinert, ${ }^{5}$ agree with each other within $0.1-0.2 \mathrm{eV}$. In the data of Collins et al. ${ }^{14}$ a similar shift away from $E_{F}$ also appears compared to their own photocurrent calculation, though the differences are less pronounced than is the case in our results. This consistent discrepancy between all these theoretical band structures and our experimental results cannot be explained by finite angular acceptance or crystal misorientation. If a nonzero $k_{\|}$is the cause of this large shift, then we should expect a large band dispersion with changing incident angle and a shift to lower energies at a certain angle. Instead, as shown in Fig. 4, tilting the crystal along either the $\langle 001\rangle$ direction or the $\langle 1 \overline{1} 0\rangle$ direction for several degrees only results in the $\Sigma_{4}$ band dispersing upward slightly. The pronounced changes in the image state also indicate that the angular resolution of the system is much better than $5^{\circ}$.

The origin of the discrepancy between the IPE experimental bands and the calculations is unclear. Several effects need to be considered, as discussed below.

(1) The one-dimensional density of states (1D DOS) could dominate the experimental spectra under certain circumstances. Most of the existing photoemission data (with a photon energy of about 25-50 eV) from clean W(110) do not show clear band dispersion with $k_{\perp}$ and are therefore considered to be more representative of the 1D DOS along the $\Gamma \Sigma N$ line, instead of the direct transition. ${ }^{10,12,13}$ This could be caused by evanescent up-

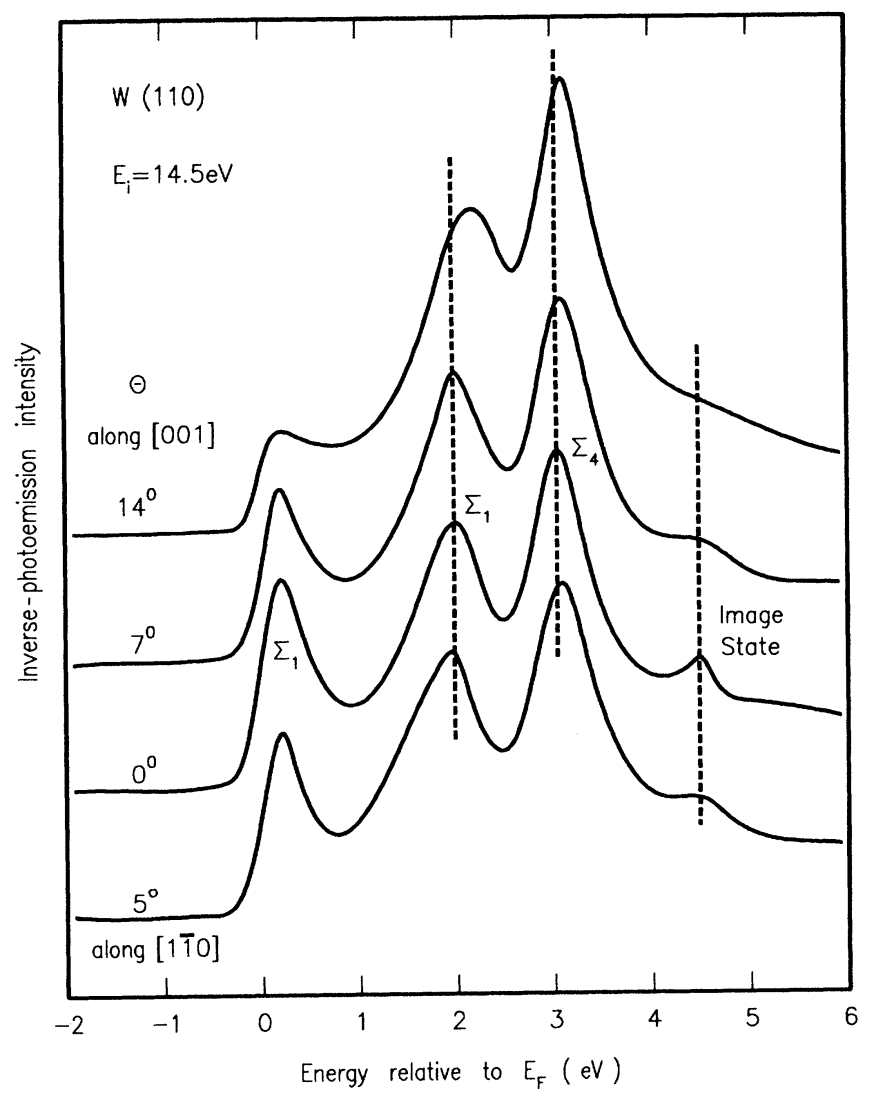

FIG. 4. Inverse-photoemission spectra of $\mathrm{W}(110)$ with different incident angles for $E_{i}=14.5 \mathrm{eV}$. The dispersion of the $\Sigma_{4}$ band is discussed in the text. The image state disperses to higher energy and becomes broader for off-normal incidence angles, as expected.

per states or by multiple upper bands. In general, we cannot assign our data to a 1D DOS since we observe dispersion for changing $k_{\perp}$ quite clearly, especially for low incidence energies. With higher incidence electron energies, there are more states along $\Gamma \Sigma N$ available (seen in the band calculation ${ }^{2}$ ) and more broadening in the initial band of IPE. It is therefore possible that the spectra become more representative of a $1 D$ DOS at higher incidence energies. This effect can result in the IPE features shifted to higher energies from $\Gamma_{12}$ and a finite apparent splitting even at the $\Gamma_{12}$ point, where the $\Sigma_{1}$ and $\Sigma_{4}$ bands should become degenerate, even in the relativistic case. Therefore we take the average between the $\Sigma_{1}$ and $\Sigma_{4}$ peaks as the $\Gamma_{12}$ point. The splitting of the IPE features at large electron energies accounts for some of the differences between experiment and theory but not all. This 1D DOS-like effect does not explain the differences that extend over a large range of the Brillioun zone, in particular for our highest $\Sigma_{4}$ point at $3.2 \mathrm{eV}$. It lies $0.4-0.5 \mathrm{eV}$ above the calculated band maximum at $N_{4}$. It would be desirable to compare photocurrent calculations, which use the real upper bands, with high-quality experimental data. The photocurrent calculation of Collins et $a l .,{ }^{14}$ however, still produces a spectrum that lies lower 
in energy than the data at higher energies (near $\Gamma$ ).

(2) Surface effects, which are not included in the bulk band calculations, ${ }^{1-5}$ can also show up in the experimental data. Feuerbacher and Christensen ${ }^{1}$ argued that the apparent shift (about $1 \mathrm{eV}$ ) of the projected gap at $\bar{\Gamma}$ of the occupied bands in their photoemission data is caused by the narrowing of the bandwidth at the surface. For the unoccupied part of the band structure, the narrowing of the surface DOS can only result in a shift toward $E_{F}$ instead of away from $E_{F}$. The existing surface density of state calculations ${ }^{6,7}$ do not reveal the presence of a surface state with an energy slightly higher than the bulk $\Sigma_{4}$ band necessary to explain the band shift to greater energies.

(3) The band-structure calculations are for the ground state of $N$ electrons while the inverse photoemission involves the excited state of $(N+1)$ electrons. This means there is always an energy difference between the experimental and theoretical band structures. This can be calculated as the real part of the self-energy term and should explain part of the discrepancy between the band calculations and experiment. Such a self-energy calculation for tungsten, however, does not exist, at present, to the authors' knowledge.

The imaginary part of the self-energy is the lifetime broadening of the excited state. For photoemission in the EDC (energy distribution curve) mode with normal emission electrons, the linewidth of the observed transition is often written as $^{21,22}$

$$
\Gamma=\left|\frac{\Gamma_{i}+\left|\frac{V_{i}}{V_{f}}\right| \Gamma_{f}}{1-\frac{V_{i}}{V_{f}}}\right|,
$$

where $\Gamma_{f}$ and $\Gamma_{i}$ are the inverse lifetimes of the electrons in final and initial states, respectively. $V_{f}$ and $V_{i}$ are the group velocities along the normal direction in the final and initial states. As Smith, Thiry, and Petroff ${ }^{23}$ recently pointed out, however, the expression of the measured linewidth is different for the different modes, i.e., EDC, CIS (constant initial state spectrum), and CFS (constant final state spectrum). For our inversephotoemission setup (using fixed incidence energy and measuring the emitted photon spectra), the expression for CFS in Ref. 22 is the suitable form:

$$
\Gamma=\Gamma_{f}+\left|\frac{V_{f}}{V_{i}}\right| \Gamma_{i},
$$

where the initial and final states are reversed as compared to the case in photoemission. While $V_{i}$ and $V_{f}$ can be measured from the initial and final band dispersion, $\Gamma_{i}$ can be estimated from the Fermi-level crossing at $11.5 \mathrm{eV}$ in Fig. 2 as about $2 \mathrm{eV}$. This corresponds to a mean free path of about $8 \AA$, which is reasonable for this energy range. By measuring the widths of the $2.0 \mathrm{eV}$ and 3.1 $\mathrm{eV}$ features in the $E_{i}=14.5 \mathrm{eV}$ spectrum and correcting for the instrumental resolution (about $0.3 \mathrm{eV}$ at these energies), we can therefore estimate the inverse lifetime of the electrons in the lower final state band as

$$
\Gamma_{f}(3.1 \mathrm{eV})=0.5 \pm 0.1 \mathrm{eV}
$$

and

$$
\Gamma_{f}(2.0 \mathrm{eV})=0.4 \pm 0.1 \mathrm{eV} .
$$

Here we have ignored the possible $k$-dependence effects. Suppose this lifetime broadening also follows a linear relationship with the energy away from $E_{F}\left[\Gamma_{f}(E)=\right.$ $\left.C\left(E-E_{F}\right)\right]$, as is the case for the $3 d$ bands of some transition metals, then the slope $C$ for the tungsten $5 d$ bands is $0.1-0.2$. This value is similar to that for single-crystal $\mathrm{Cu}(0.1),{ }^{22}$ but much lower than that for Fe (0.6) ${ }^{24}$ Given that there exist other sources of peak broadening, such as phonon contribution, ${ }^{11}$ surface imperfections, etc., the values given here should be considered as the upper bounds of the intrinsic lifetime broadening.

By taking luminescence data at different high incident electron energies (for example, $40 \mathrm{eV}$ ), several constant photon energy features are revealed at $10.3 \mathrm{eV}$, $14.5 \mathrm{eV}$, and $20.8 \mathrm{eV}$. These features are consistent with the known surface-plasmon modes of $9.7 \mathrm{eV}, 14.8 \mathrm{eV}$, and $20.8 \mathrm{eV}$, as obtained by optical measurements. ${ }^{25} \mathrm{Al}$ though some of these peaks are in the neighborhood of the Fermi-level crossing points, it is clear that the bands indeed cross $E_{F}$ around 11.5 and $20.5 \mathrm{eV}$ by taking the corresponding plot of intensity versus photon energy for final states above $E_{F}$.

\section{CONCLUSION}

The unoccupied tungsten $5 d$ band structure has been mapped out with a three-step direct transition model. The discrepancy between the experimental results and the band calculations has been discussed, particularly in terms of the real part of the self-energy, and the onedimensional density of state effects. The upper bound of the energy-dependent lifetime broadening is estimated as $0.4 \mathrm{eV}$ at $2.0 \mathrm{eV}$ above $E_{F}$ and $0.5 \mathrm{eV}$ at $3.1 \mathrm{eV}$ above $E_{F}$.

\section{ACKNOWLEDGMENTS}

D.L. and P.A.D. were partially supported by Syracuse University and the NSF (Grant Nos. DMR-88-20779 and DMR-92-21655).
${ }^{1}$ N. E. Christensen and B. Feuerbacher, Phys. Rev. B 10, 2349 (1974); B. Feuerbacher and N. E. Christensen, ibid. 10, 2373 (1974).

${ }^{2}$ R. F. Willis and N. E. Christensen, Phys. Rev. B 18, 5140 (1978); N. E. Christensen and R. F. Willis, J. Phys. C 12,
167 (1979).

${ }^{3}$ D. M. Bylander and L. Kleinman, Phys. Rev. B 29, 1534 (1984).

${ }^{4}$ H. J. F. Jansen and A. J. Freeman, Phys. Rev. B 30, 561 (1984). 
${ }^{5}$ S.-H. Wei, H. Krakauer, and M. Weinert, Phys. Rev. B 32, $7792(1985)$.

${ }^{6}$ O. Jepsen and R. O. Jones, Phys. Rev. B 34, 6695 (1986).

${ }^{7}$ M. P. López Sancho, J. M. López Sancho, and J. Rubio, J. Phys. C 18, 1803 (1985).

${ }^{8}$ W. Drube, D. Straub, F. J. Himpsel, P. Soukiassian, C. L. Fu, and A. J. Freeman, Phys. Rev. B 34, 8989 (1986).

${ }^{9}$ M. W. Holmes, D. A. King, and J. E. Inglesfield, Phys. Rev. Lett. 42, 394 (1979).

${ }^{10}$ R. H. Gaylord, K. H. Jeong, and S. D. Kevan, Phys. Rev. Lett. 62, 2036 (1989); Phys. Rev. B 36, 9337 (1987); 37, 8491 (1988).

${ }^{11}$ S. Dhar and S. D. Kevan, Phys. Rev. B 41, 8516 (1989).

${ }^{12}$ R. C. White, C. S. Fadley, M. Sagurton, and Z. Hussain, Phys. Rev. B 34, 5226 (1986).

${ }^{13}$ G. B. Blanchet, N. J. DiNardo, and E. W. Plummer, Surf. Sci. 118, 496 (1982).

${ }^{14}$ I. R. Collins, A. D. Laine, P. T. Andrews, and P. J. Durham, J. Phys. Condens. Matter 3, 5307 (1991); 4, 2891 (1992).

${ }^{15}$ D. Funnemann and H. Merz, J. Vac. Sci. Technol. A 5, 657 (1987).
${ }^{16}$ I. L. Krainsky, J. Vac. Sci. Technol. A 5, 735 (1987).

${ }^{17}$ P. O. Nilsson and C. G. Larsson, Jpn. J. Appl. Phys. Suppl. 17, 144 (1978).

${ }^{18}$ A. Horlacher-Smith, R. A. Barker, and P. J. Estrup, Surf. Sci. 136, 329 (1984); L. D. Roelofs, J. W. Chung, S. C. Ying, and P. J. Estrup, Phys. Rev. B 33, 6537 (1986).

${ }^{19}$ For example, R. G. Musket, W. McLean, C. A. Colmenowes, D. M. Mako Wiezcki, and W. J. Siekhaus, Appl. Surf. Sci. 10, 143 (1982); Dongqi Li, Jiandi Zhang, P. A. Dowben, and M. Onellion, Phys. Rev. B 45, 7272 (1992).

${ }^{20}$ R. F. Girvan, A. V. Gold, and R. A. Phillips, J. Phys. Chem. Solids 29, 1485 (1968).

${ }^{21}$ T. -C. Chiang, J. A. Knapp, M. Aono, and D. E. Eastman, Phys. Rev. B 21, 3513 (1980).

${ }^{22}$ J. A. Knapp, F. J. Himpsel, and D. E. Eastman, Phys. Rev. B 19, 4952 (1979).

${ }^{23}$ N. V. Smith, P. Thiry, and Y. Petroff (unpublished).

${ }^{24}$ A. Santoni and F. J. Himpsel, Phys. Rev. B 43, 1305 (1991).

${ }^{25} \mathrm{~J}$. H. Weaver, C. G. Olson, and D. W. Lynch, Phys. Rev. B 12, 1293 (1975). 